\title{
Reentanglement Kinetics in Polyisobutylene
}

\author{
D. Roy ${ }^{\dagger}$ and C. M. Roland* \\ Chemistry Division, Naval Research Laboratory, Code 6120, Washington, D.C. 20375-5342, United States
}

ABSTRACT: The rheological properties of a polyisobutylene (PIB) having a molecular weight five times its entanglement molecular weight were measured, along with interrupted shear flow experiments to quantify the time required for structural recovery following steady state shearing in the non-Newtonian regime. The reentanglement kinetics was substantially slower (by more than an order of magnitude) than expectations based on the linear relaxation time of the fully entangled material. This result is in accord with published interrupted shear flow results for concentrated polymer solutions. The retarded recovery of entanglements may underlie other anomalies, for example, the delayed development of autoadhesion (tack) in rubber, the behavior of freeze-dried dilute solutions and the limiting behavior of the molecular weight dependence of the viscosity. It also offers a route to more processable polymers and, being reversible, will not affect the ultimate physical properties.

\section{INTRODUCTION}

When a polymer melt or solution is sheared at rates sufficiently low that the perturbation of the chains is slow compared to the rate of Brownian motion, their equilibrium conformation is retained, and the viscosity is Newtonian. Shear rates exceeding the reciprocal of the linear relaxation time, however, induce chain stretching and a reduction of entanglements, causing a decrease of the steady-state viscosity, $\eta_{s s}$ ("shear-thinning"). ${ }^{1-3}$ During the initial phase of non-Newtonian flow, the stress and normal force both pass through a maximum versus time, as the chains disentangle prior to attaining steady state; ${ }^{4}$ birefringence measurements reveal similar behavior. ${ }^{5}$ The magnitude of the overshoot peak increases with shear rate, occurring at strains around 2 to $3^{2}$ with some increase at high rates. ${ }^{4}$ Such behavior is characteristic of a viscoelastic material with time-dependent structure (entanglements in this case), although unentangled polymers can also exhibit stress overshoots and shear thinning, but the effects are weaker. ${ }^{6,7}$

Herein we study reentanglement kinetics using interrupted non-Newtonian shear flow experiments, in which after steady state is achieved, the flow is halted and the material held in a quiescent state for some time before resumption of the shearing. Since the flow-induced loss of entanglements is reversible, the magnitude of the stress overshoot during subsequent shearing has an amplitude governed by the time allowed for reentanglement. ${ }^{4,8-15}$ The steady-state viscosity is unaffected by interruption of the flow, since it depends only on the shear rate. For rest times exceeding some characteristic time, the entanglements return to their equilibrium concentration, and the magnitude of the overshoot has its maximum (initial) value. For concentrated solutions, the time to recover the fully entangled state has been shown to have the same molecular weight dependence $\left(c a . M^{3.4}\right)$ as the viscosity and

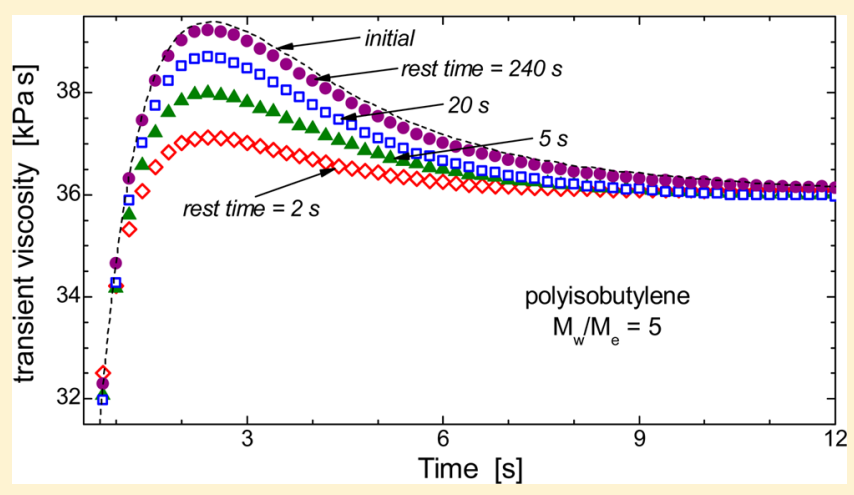

linear relaxation time. ${ }^{16}$ Herein we determine its dependences on temperature and shear rate for a polymer melt.

Although the flow behaviors of concentrated polymer solutions and neat melts are similar, transient measurements can be problematic for the latter because their greater elasticity makes experiments at non-Newtonian rates more susceptible to artifacts, due inter alia to inhomogeneous flow (shear rate discontinuities within the flowing material $)^{17-20}$ or fracture and loss of material at the edge. ${ }^{21}$ For this reason interrupted shear flow studies of entangled polymers to date have been carried out on solutions, ${ }^{4,5,8-10,14-16}$ with the exception of two studies ${ }^{11,12}$ on commercial, semicrystalline polyethylene resins. The latter had broad molecular weight distributions and branching, which make difficult the determination of the linear viscoelastic response in the terminal regime. In this work we performed interrupted shear flow experiments on neat polyisobutylene (PIB). The molecular weight was five times the entanglement molecular weight of PIB $\left(M_{e}=9400 \mathrm{Da}^{22}\right)$, sufficiently high to yield stress overshoots but low enough that melt fracture and inhomogeneous flow could be avoided. We find that consistent with prior results on polymer solutions, the entanglement recovery time is significantly longer than the linear relaxation time; that is, partially disentangled chains recover their structure more slowly than expected from their equilibrium mobility, even though diffusion per se is faster for chains having fewer entanglements. This retarded structural recovery is observed for various shear rates and temperatures. Unexpectedly slow reentanglement may underlie other anomalies in the polymer literature, including the behavior of

Received: October 8, 2013

Revised: November 13, 2013

Published: November 19, 2013 
freeze-dried solutions and the tack of rubber. The practical significance is that flow-induced modification offers a means to improve the processability of a polymer by reducing the viscosity and elasticity. But unlike conventional methods to accomplish this, flow modification is reversible and thus does not affect physical properties such as the strength and hysteresis.

\section{EXPERIMENTAL SECTION}

The polyisobutylene (Oppanol B11 from BASF) had a weight-average molecular weight of $46000 \mathrm{~g} / \mathrm{mol}\left(M_{\mathrm{w}} / M_{e}=5\right)$, with a polydispersity $=3.2$. Dynamic shear measurements were carried out with an Anton Paar MCR 502 rheometer using a parallel plate geometry. The frequency range was $0.001 \leq \omega(\mathrm{rad} / \mathrm{s}) \leq 100$, and temperatures were from -15 to $90{ }^{\circ} \mathrm{C}$. Steady state and transient shear measurements were done using a cone and the plate geometry $\left(8 \mathrm{~mm}\right.$ diameter; $0.9^{\circ}$ cone angle). The interrupted shear flow experiments were done at shear rates of from 0.3 to $1.0 \mathrm{~s}^{-1}$, at temperatures over the range 25 to $45{ }^{\circ} \mathrm{C}$.

\section{RESULTS}

Linear Viscoelastic Properties. Figure 1 displays master curves of the dynamic storage, $G^{\prime}$, and loss, $G^{\prime \prime}$, moduli for the

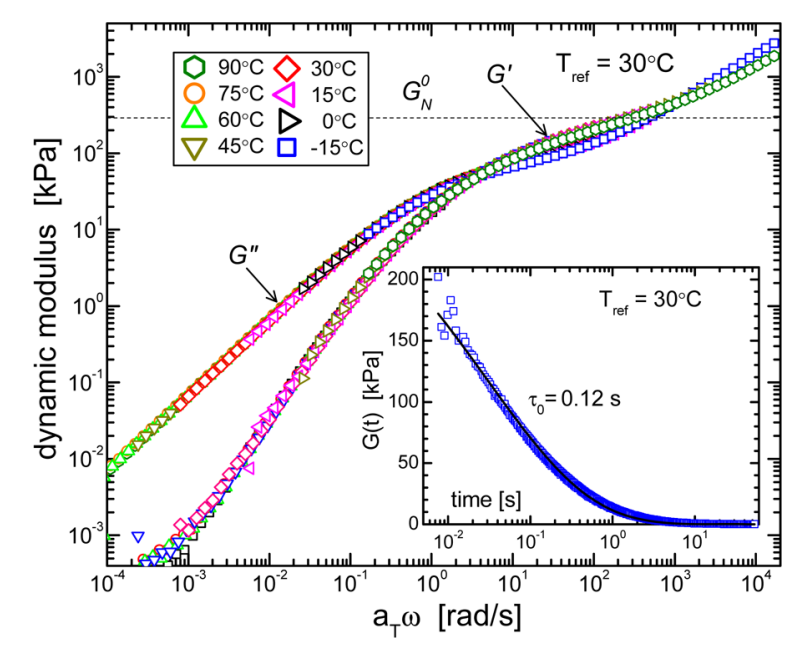

Figure 1. Master curves of the dynamic shear moduli; the shift factors, plotted in Figure.2, were determined from superposition of the loss tangent. The plateau modulus from ref $^{22}$ is indicated by the horizontal dotted line. The inset shows the stress relaxation modulus master curve, obtained using the same shift factors, with the solid line the fit to eq 3

PIB. The temperature-frequency shift factors, $a_{T}$, used to construct these plots were determined by superposing the loss tangent, since that requires no vertical shifting. There is only a very weak plateau in $G^{\prime}$ and no discernible maximum in $G^{\prime \prime}$, reflecting the low degree of chain entanglement. (The plateau modulus determined for higher molecular weight PIB, $G_{N}^{0}=$ $290 \mathrm{kPa}^{22}$ is indicated in the figure.) These data only encompass the chain dynamics, and as seen in Figure 2, the $a_{T}$ exhibit an essentially Arrhenius temperature dependence over the 105 degree range of the measurements. The apparent activation energy, $E_{a}=69 \mathrm{~kJ} / \mathrm{mol}$, is equivalent to that reported by Wang and Porter. ${ }^{23}$ Included in the figure are literature viscosity data ${ }^{22}$ for a higher molecular weight PIB, which show some deviation from Arrhenius behavior.

In Figure 3 are the viscosities, including both steady state as a function of shear rate and dynamic measurements as a function

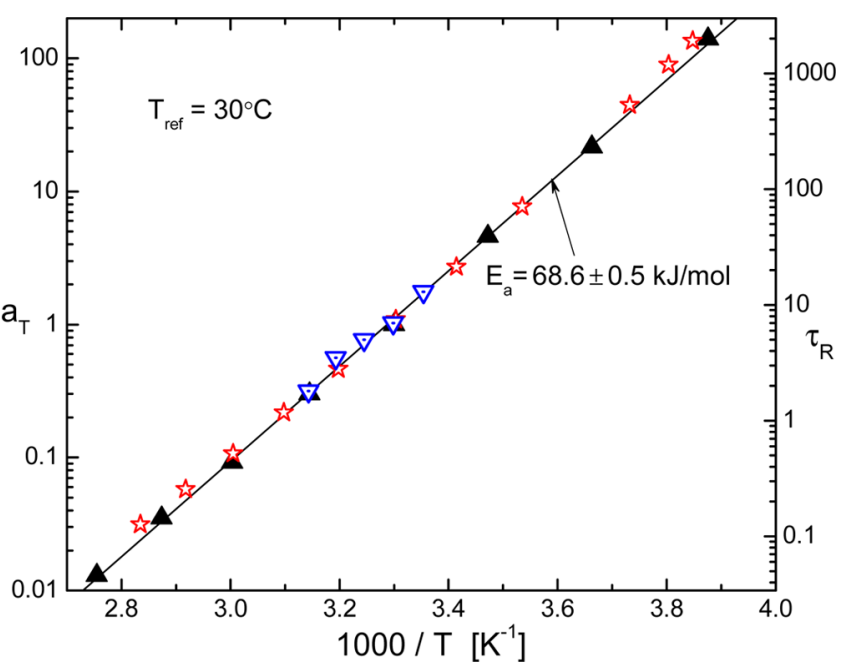

Figure 2. Shift factors used to construct Figure 1 (filled triangles) and the reentanglement times determined from interrupted shear flow experiments (inverted triangles), showing their equivalent temperature dependences. Also included are shift factors for a higher molecular weight PIB $\left(M_{\mathrm{w}}=176 \mathrm{~kg} / \mathrm{mol}\right.$; open stars $)$, which show some deviation from Arrhenius behavior. ${ }^{22}$

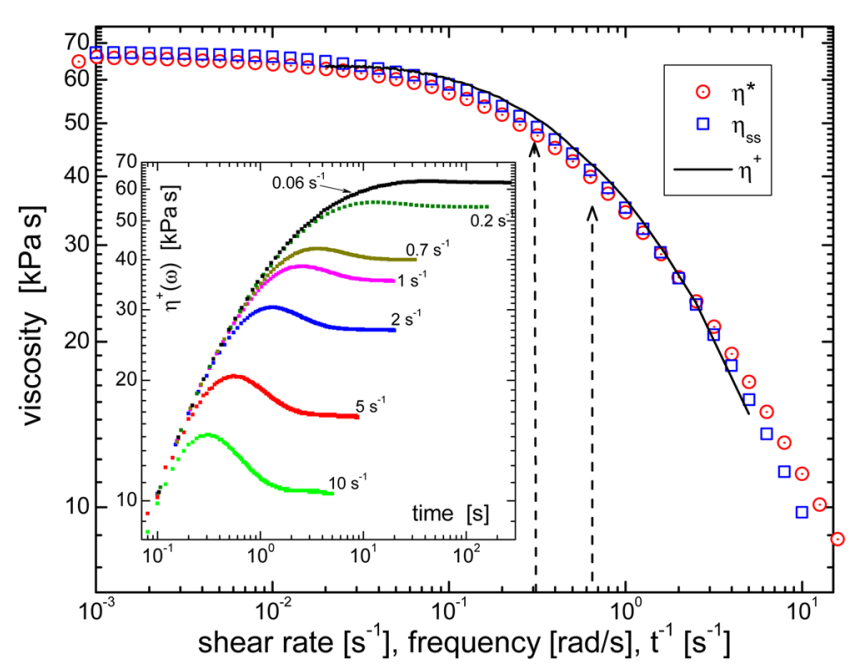

Figure 3. Steady-state shear viscosity (squares), dynamic viscosity (circles), and transient viscosity (solid line), the latter determined at low shear rate using eq 2 . The vertical arrows denote the range of shear rates used for the overshoot recovery experiments. The inset shows the transient viscosity measured during start-up shear flow at different shear rates; shear-thinning behavior is associated with appearance of a stress overshoot. All measurements were at $30^{\circ} \mathrm{C}$.

of frequency. The latter are a few percent larger than the former; for example in the Newtonian regime $\lim _{\omega \rightarrow 0} \eta^{*}(\omega)=65.8$ $\mathrm{kPa}$ s and $\lim _{\dot{\gamma} \rightarrow 0} \eta_{S S}(\dot{\gamma})=67.2 \mathrm{kPa}$ s. This essential conformance to the Cox-Merz relation ${ }^{24}$

$$
\left|\eta^{*}(\omega)\right|_{\dot{\gamma}=\omega}=\eta_{s s}(\dot{\gamma})
$$

affirms the integrity of the steady state measurements; that is, an absence of flow inhomogeneities or edge effects. Also plotted in Figure 3 is the transient viscosity measured at low shear rate $\left(=0.06 \mathrm{~s}^{-1}\right)$, with the abscissa values taken as the reciprocal of the measurement time. The equivalence with the steady shear viscosity is known as the Gleissle relation ${ }^{25}$ 


$$
\left.\lim _{\dot{\gamma} \rightarrow 0} \eta^{+}(t, \dot{\gamma})\right|_{t^{-1}=\dot{\gamma}}=\eta_{s s}(\dot{\gamma})
$$

The range of shear rates used in the interrupted shear flow experiments (described below) is denoted in Figure 3 by the vertical lines. The onset of non-Newtonian behavior is associated with flow-induced disentanglement.

Relaxation Time. It is customary to determine a relaxation time as the inverse of the frequency of the terminal peak in the loss modulus. However, because of the low degree of entanglements for the PIB herein, the maximum in $G^{\prime \prime}$ is negligible. A characteristic time constant, $\tau_{x}$, can be defined from the frequency at which the storage and loss moduli are equal, a crossover of $G^{\prime}$ and $G^{\prime \prime}$ only occurring for entangled polymers. $^{26}$ This quantity is ill-defined, being affected by polydispersity, and thus provides only an estimate of the terminal relaxation time; from Figure $1 \tau_{x}=0.21 \mathrm{~s}$.

The stress relaxation modulus measured for the material is shown in the inset to Figure 1 . At $30{ }^{\circ} \mathrm{C}$ the stress decays to a negligible value (on a linear ordinate) in less than $10 \mathrm{~s}$. A function can be fit to the data to obtain a time constant that reflects an average relaxation time. For example, the Kohlrausch stretched exponential can be used ${ }^{27,28}$

$$
G(t)=G_{N}^{0} \exp \left[-\left(t / \tau_{0}\right)^{\beta}\right]
$$

where $\tau_{0}$ is a relaxation time, and the stretch exponent has values in the range $0<\beta \leq 1$. Applying eq 3 to the stress relaxation data yields $\tau_{0} \sim 0.12 \mathrm{~s}$ (with $G_{N}^{0}=250 \mathrm{kPa}$ and $\beta=$ $0.6)$; this is consistent with the magnitude of $\tau_{x}$. The longest relaxation time for a viscoelastic material is sometimes obtained as the inverse of the limiting slope of a plot of $\log G(t)$ versus linear time, which assumes the relaxation time distribution can be described by a sum of simple exponential decay. Instead we define a maximum relaxation time, $\tau_{\max }$, as the time for $G(t)$ to relax to $1 \%$ of $G_{N}^{0}$ (note the segmental dynamics begin to encroach at the shortest short times in Figure 1). This definition is model-independent, albeit arbitrary. At $30{ }^{\circ} \mathrm{C}$, we find $\tau_{\max }=3.7 \mathrm{~s}$. The span of $\tau_{0}$ and $\tau_{\max }, 1.5$ decades, reflects the breadth of the terminal relaxation dispersion for this moderately polydisperse polymer.

The linear relaxation time of the PIB corresponds to the time scale for the fully entangled chains to move a distance commensurate with their size. This quantity is a linear property because the perturbation used in the experimental determination does not drive the system further from equilibrium than the fluctuations prevailing in the unperturbed material. However, strain rates sufficient to reduce the viscosity from its Newtonian value are nonlinear, and hence the characteristic time scales in shear thinning experiments presumably differ from linear relaxation times. The issue is whether reentanglement requires longer times (as previous work on polymer solutions indicates ${ }^{4,5,8-10,14-16}$ ) or shorter (as expected for chains that are less entangled).

Interrupted Shear Flow. The shear-thinning phenomenon is illustrated in the inset to Figure 3, showing the timedependent viscosity, $\eta^{+}(t, \dot{\gamma})$, after imposition of shearing at various rates. With increasing $\dot{\gamma}$, the transient viscosity goes through a maximum that occurs at a roughly constant value of the strain $(=\dot{\gamma} t)$ equal to $2.6 \pm 0.2$; this result is consistent with literature values. ${ }^{2,4,6,29}$ The magnitude of the overshoot peak increases with shear rate due to greater chain anisotropy and more entanglement reduction, in turn giving rise to smaller steady state viscosities.
If the flow is stopped then restarted, the magnitude of the overshoot is smaller. It increases with the duration of the rest time, as chains reentangle during the quiescent period, with the original amplitude recovered for sufficiently long rest periods (Figure 4). The kinetics of the reentangling are illustrated in

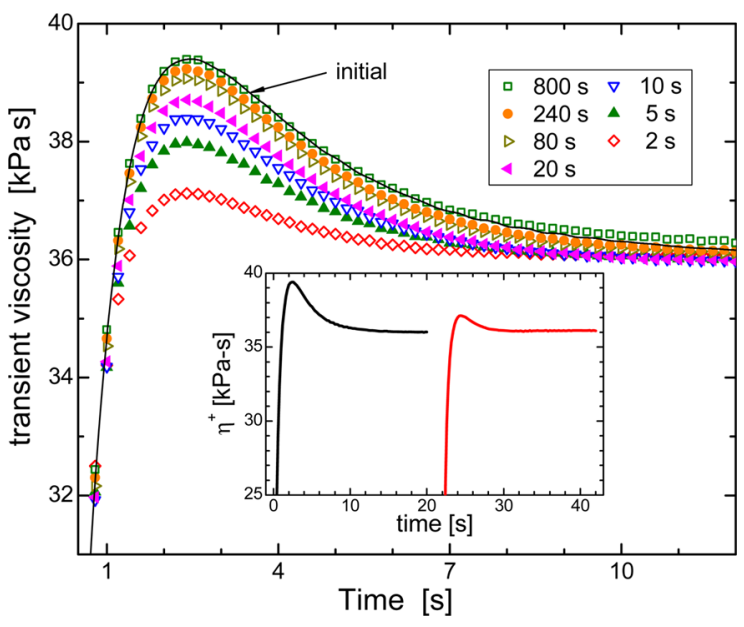

Figure 4. Interrupted transient viscosity measured after the rest period indicated in the legend. The inset illustrates the experiment for a $2 \mathrm{~s}$ rest period. The shear rate was $1.0 \mathrm{~s}^{-1}$ and $\mathrm{T}=30^{\circ} \mathrm{C}$.

Figure 5, showing the magnitude of the stress overshoot versus recovery time for various shear rates at $T=30{ }^{\circ} \mathrm{C}$. The overshoot recovery data can be described using a stretched exponential function

$$
\eta_{\max }^{+}=\eta_{s s}+A\left(1-\exp \left[-\left(t / \tau_{R}\right)^{\beta}\right]\right)
$$

This form has been applied previously to terminal relaxation of long chain polymers, with $\beta \sim 0.6$ for monodisperse polymers. ${ }^{28,30}$ In eq $4 A$ is a constant and $\tau_{\text {rec }}$ is a characteristic time to recover the state of entanglement associated with no flow. Fitting this expression to the measurements at $30{ }^{\circ} \mathrm{C}$, we obtain $\tau_{\text {rec }}=7.0 \pm 1.0 \mathrm{~s}$, independent of shear rate over the range from 0.3 to $1 \mathrm{~s}^{-1}$; that is, the characteristic time for entanglement recovery is independent of the degree of disentanglement, at least over this range of shear rates. The recovery time varied inversely with temperature; these data, obtained over the range 25 to $45^{\circ} \mathrm{C}$ for $\dot{\gamma}=1 \mathrm{~s}^{-1}$, are included in Figure 2. As can be seen, the activation energy for reentanglement is equivalent to that for the time-temperature shift factors for the linear dynamic shear modulus (Figure 1).

\section{DISCUSSION}

Flow inhomogeneities or discontinuities due to slipping at the interface can cause stress overshoots qualitatively similar to those in Figure 4. A direct indication that these are not occurring in the present experiments is the good agreement between the dynamic and steady state viscosities (Figure 1). Boukany et al. ${ }^{31}$ reported slipping occurred at a critical stress of 0.1-0.2 $\mathrm{MPa}$; the highest stresses in our interrupted flow measurements were about an order of magnitude smaller. The apparent absence of any flow discontinuities herein is consistent with Ravidranath et al., ${ }^{32}$ who found that shear banding in steady shear flow required higher molecular weight polymers. For example, 1,4-polybutadiene with as many as 13 entanglements per chain exhibited homogeneous flow under all conditions; $^{32}$ for the PIB in this work $M_{w} / M_{e}=5$. 


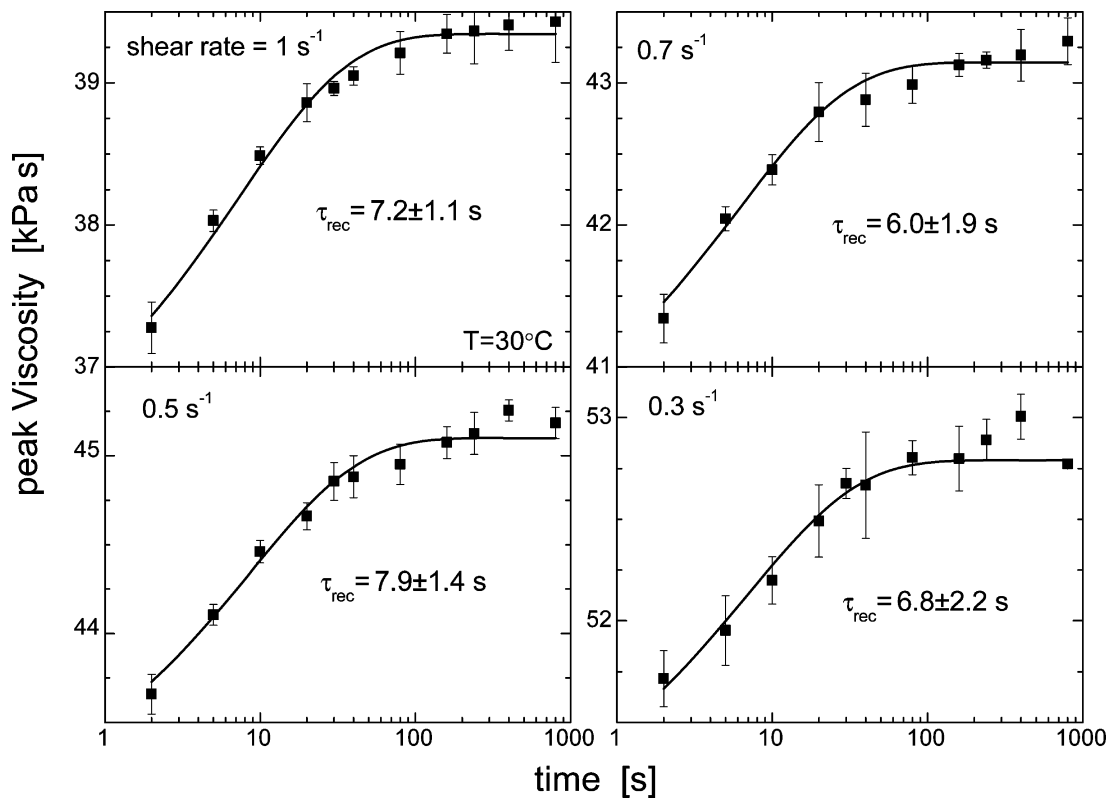

Figure 5. Maximum in the transient viscosity as a function of recovery time for various shear rates at the indicated temperature.

Table 1 compares two linear relaxation times-from fitting eq 3 to the stress decay data and the time for $99 \%$ relaxation of

Table 1. Linear Relaxation Times and Recovery Times for PIB at Different Temperatures

$\begin{array}{cllc}T\left({ }^{\circ} \mathrm{C}\right) & \tau_{0}(\mathrm{~s}) & \tau_{\max }(\mathrm{s})^{a} & \tau_{\text {rec }}(\mathrm{s}) \\ 25 & 0.20 & 6.3 \pm 0.7 & 13 \pm 5.0 \\ 30 & 0.12 & 3.7 \pm 0.2 & 7.0 \pm 1.0 \\ 35 & 0.079 & 2.5 \pm 0.3 & 5.0 \pm 1.0 \\ 40 & 0.050 & 1.6 \pm 0.1 & 3.5 \pm 0.3 \\ 45 & 0.035 & 1.1 \pm 0.1 & 1.8 \pm 0.3\end{array}$

${ }^{a}$ Time for $99 \%$ of stress to relax.

the stress-to the recovery time from eq 4. Both linear relaxation times are substantially smaller than $\tau_{\text {rec }}$ which negates any argument based on polydispersity or the definition of relaxation time for the disparity with $\tau_{\text {rec }}$. The anomalously slow entanglement recovery is found for all shear rates and temperatures (Table 1 and Figure 5). Interestingly $E_{a}$ for the reentanglement kinetics is equal to the value of the apparent activation energy for the Newtonian viscosity of higher molecular weight $\mathrm{PIB}^{23}$ (Figure 2).

Entanglement recover transpiring over a longer time scale than the linear relaxation of stress has been previously reported for concentrated polymer solutions; these results are tabulated in Table 2. We believe the general finding of unexpectedly slow reentanglement kinetics underlies anomalies in the polymer rheology literature. In adhesion ("tack") experiments on uncured rubber, the assumption is that full entanglement across a perfectly formed interface requires diffusion of the chain over a distance equal to its size, a time scale reflected in $\tau_{0}{ }^{33}$ However, the time required for development of adhesion in such experiments has been found to be substantially longer than the time calculated from the linear diffusion constant. ${ }^{33,34}$ Another manifestation of retarded reentanglement is the equilibration time after removal of the solvent from polymer solutions. Colby et al. ${ }^{35}$ reported that a 1,4-polybutadiene with a $M_{\mathrm{w}}$ exceeding 16 million dalton, for which the terminal
Table 2. Stress Overshoot Recovery Times for Various Polymers

\begin{tabular}{lccl} 
& $\eta_{\max } / \eta_{s s}$ & $\tau_{\text {rec }} / \tau_{0}$ & reference \\
polyisobutylene & 1.1 & 58 & this work \\
polybutadiene solutions & 1.3 & 30 & 10,16 \\
polystyrene solutions & $>2$ & $\sim 2$ & 14 \\
polysaccharide in water & $\sim 1.2$ & $>10$ & 15 \\
polyethylene & - & $>100$ & 12 \\
polyethylene & 1.2 & $\sim 10$ & 11,13 \\
polybutadiene solutions & $\sim 1.6$ & $\sim 10$ & 4 \\
polystyrene solutions & 16 & $\sim 10$ & 8 \\
\hline
\end{tabular}

relaxation issue was calculated to be 4.4 days, had not attained an equilibrium state even one month after drying. This example is especially significant since the viscosity measured for that sample, presumably lower than the equilibrium value, was used to confirm the $\eta_{0} \propto M^{3}$ theoretical prediction for the molecular weight dependence. A related observation has been made for freeze-dried solutions. Viscosity ${ }^{36,37}$ and dynamic mechanical ${ }^{38}$ measurements on melts prepared by freeze-drying are consistent with reduced chain entanglements, persisting from the original dilute solution from which the solvent had been sublimed. The recovery of entanglements observed for freezedried polymer melts is highly retarded, even though small-angle neutron scattering on freeze-dried polymers shows the chains to have their normal size (no "compaction"), ${ }^{39}$ and solid state ${ }^{13} \mathrm{C}$ NMR dipolar dephasing measurements show the chains to be at least partially interspersed. ${ }^{40,41}$ There are likely other unexplained phenomena in polymer rheology having their origin in the fact that entanglements reform more slowly than suggested by the linear diffusion constant.

What causes the structural recovery of entangled polymers to be slow? Convective constraint release accelerates relaxation during flow, although this effect is absent for the quiescent state. ${ }^{14}$ Nevertheless, the direct effect of flow, entanglement loss, should accelerate relaxation and subsequent structural recovery; that is, diffusive processes should not be slower for partially unentangled chains than for the corresponding fully entangled condition. Note in Table 2 that there is no 
correlation between the ratio $\tau_{r e c} / \tau_{0}$ and the magnitude of the stress overshoot peak relative to the steady state stress (although for recovery of the weak stress overshoots in unentangled polystyrene, $\tau_{0}=\tau_{\text {rec }}{ }^{6}$ ). This observation is consistent with the absence of any significant effect of shear rate on $\tau_{\text {rec }}$ (Figure 5). The disconnects between the linear relaxation time and the reentanglement time $\left(\tau_{\text {rec }}>\tau_{0}\right)$ and between the reentanglement kinetics and the degree of disentanglement are evidently related to the fact that, after nonlinear flow, neighboring chains are not fully entangled, whereby motion over distances commensurate with the chain size does not lead to full recovery. The reentanglement time scale is greater than the linear relaxation time, notwithstanding the enhancement of the diffusion constant due to loss of entanglements, because structural recovery involves interdigitation among chains having growing degrees of entanglement. The underlying mechanism is stochastic displacements of chain segments, the same as for equilibrium diffusion, as reflected in the equivalence of the activation energies for $\tau_{r e c}$ and the linear viscoelastic shift factors (Figure 2).

The implication of retarded recovery of the fully entangled state is that nonlinear flow can be used to enhance the processability of polymers. The resistance to flow is reduced, making processing more efficient. The slow recovery of the elasticity (e.g., normal stresses) means that shear modification can be used to reduce extrudate (die) swell and related properties, and molding operations may require less time to achieve shape retention. Since the shear-modified condition is reversible, physical properties such as a polymer's stiffness and strength are unaffected. The usual methods of facilitating processing, such as lowering the molecular weight or the use of plasticizers and diluents, reduce ultimate performance. This would not be the case for shear modification, provided sufficient annealing occurs prior to curing in order to avoid a permanently nonequilibrium structure.

\section{SUMMARY}

Interrupted shear flow experiments were carried out on a neat polyisobutylene having a molecular weight 5 times the entanglement molecular weight. Our main findings are as follows:

1 In accord with previous studies, mainly on concentrated polymer solutions, the time required for reentanglement following steady state shear flow at non-Newtonian rates was significantly longer than the characteristic time associated with linear stress relaxation of the fully entangled PIB. The substantial difference between these two time constants (more than an order of magnitude) implies that subsequent to deformation, the properties of a polymeric liquid may depend on the prior history, even though the stress has relaxed to zero; that is, mechanical equilibrium defined as the absence of external stress does not necessarily correspond to structural equilibrium. This retarded entanglement recovery can be exploited to facilitate processing.

2 The kinetics of entanglement recovery in the quiescent PIB was not measurably affected by the degree of disentanglement; that is, the recovery did not depend on the prior shear rate. This anomaly is not accounted for by models of the rheology of entangled polymers.

3 Notwithstanding the disconnect between the reentanglement kinetics and linear relaxation behavior, the temperature dependence of $\tau_{0}$ and $\tau_{\text {rec }}$ are the same. Over the $20 \mathrm{~K}$ range measured herein, the stress overshoot recovery is characterized by an $E_{a}=68.6 \pm$ $0.5 \mathrm{~kJ} / \mathrm{mol}$, which is in accord with the temperature dependence of the linear time-temperature shift factors for the present polymer, and with viscosity of higher molecular weight PIB. Prior work on 1,4-polybutadiene solutions found that the molecular weight dependence of the entanglement recovery time was similarly unaffected by flow-induced disentanglement, having the usual 3.4power dependence on chain length. ${ }^{10}$

\section{AUTHOR INFORMATION}

\section{Corresponding Author}

*E-mail: (C.M.R.) roland@nrl.navy.mil.

\section{Notes}

The authors declare no competing financial interest.

${ }^{\dagger}$ National Research Council/Naval Research Laboratory postdoctoral fellow

\section{ACKNOWLEDGMENTS}

This work was supported by the Office of Naval Research. D.R. acknowledges a National Research Council/Naval Research Laboratory postdoctoral fellowship.

\section{REFERENCES}

(1) Graessley, W. W. Adv. Polym. Sci. 1974, 16, 1-179.

(2) Watanabe, H. Prog. Polym. Sci. 1999, 24, 1253-1403.

(3) Wang, S.-Q.; Wang, Y.; Cheng, S.; Li, X.; Zhu, X.; Sun, H. Macromolecules 2013, 46, 3147.

(4) Menezes, E. V.; Graessley, W. W. J. Polym. Sci., Polym. Phys. Ed. 1982, 20, 1817-1833.

(5) Oberhauser, J. P.; Leal, L. G.; Mead, D. W. J. Polym. Sci. Polym. Phys. Ed. 1998, 36, 265-280.

(6) Santangelo, P. G.; Roland, C. M. J. Rheol. 2001, 45, 583-594.

(7) Schweizer, T. Rheol. Acta 2007, 46, 629-637.

(8) Stratton, R. A.; Butcher, A. F. J. Polym. Sci. Polym. Phys. Ed. 1973, $11,1747-1758$

(9) Huppler, J. D.; MacDonald, I. F.; Ashare, E.; Spriggs, T. W.; Bird, R. B.; Holmes, L. A. J. Rheol. 1967, 11, 181-204.

(10) Robertson, C. G.; Warren, S.; Plazek, D. J.; Roland, C. M. Macromolecules 2004, 37, 10018-10023.

(11) Dealy, J. M.; Tsang, W. J. Appl. Polym. Sci. 1981, 26, 11491158.

(12) Yamaguchi, M.; Gogos, C. G. Adv. Polym. Tech. 2001, 20, 261269.

(13) Xu, Y. Z.; Dekee, D.; Fong, C. F. C. M. J. Appl. Polym. Sci. 1995, $55,779-786$.

(14) Sanchez-Reyes, J.; Archer, L. A. J. Rheol. 2003, 47, 469-482.

(15) Richardson, R. K.; Ross-Murphy, S. B. Int. J. Biol. Macro. 1987, 9, 250-256.

(16) Roland, C. M.; Robertson, C. G. Rubber Chem. Technol. 2006, $79,267-280$.

(17) Tapadia, P.; Wang, S.-Q. Macromolecules 2004, 37, 9083-9095.

(18) Schweizer, T. Rheol. Acta 2007, 46, 629-637.

(19) Manneville, S. Rheol. Acta 2008, 47, 301-318.

(20) Moorcroft, R. L.; Fielding, S. M. Phys. Rev. Lett. 2013, 110, 086011.

(21) Inn, Y. W.; Wissbrun, K. F.; Denn, M. M. Macromolecules 2005, 38, 9385-9388.

(22) Santangelo, P. G.; Roland, C. M.; Puskas, J. E. Macromolecules 1999, 32, 1972-1977.

(23) Wang, J. S.; Porter, R. S. Rheol. Acta 1995, 34, 496-503.

(24) Cox, W. P.; Merz, E. H. J. Polym. Sci. 1958, 28, 619-622.

(25) Gleissle, W. In Rheology; Astarita, G., Marucci, G., Nicolais, L., Eds.; Plenum: New York, 1980, Vol. 2, pp 457-462. 
(26) Ferry, J. D. Viscoelastic Behavior of Rubbery Materials: Wiley: New York, 1980.

(27) Narkis, M.; Hopkins, I. L.; Tobolsky, A. V. Polym. Eng. Sci. 1970, $10,66-69$.

(28) Knoff, W. F.; Hopkins, I. L.; Tobolsky, A. V. Macromolecules 1971, 6, 750-754.

(29) Meissner, J. J. Appl. Polym. Sci. 1972, 16, 2877-2899.

(30) Santangelo, P. G.; Ngai, K. L.; Roland, C. M. Polymer 1998, 39, 681-687.

(31) Boukany, P. E.; Tapadia, P.; Wang, S.-Q. J. Rheol. 2006, 50, 641-654.

(32) Ravindranath, S.; Wang, S.-Q.; Olechnowicz, M.; Quirk, R. P. Macromolecules 2008, 41, 2663-2670.

(33) Roland, C. M. Viscoelastic Behavior of Rubbery Materials; Oxford: Oxford, U.K., 2012.

(34) Roland, C. M.; Bohm, G. G. A. Macromolecules 1985, 18, 13101314.

(35) Colby, R. H.; Fetters, L. J.; Graessley, W. W. Macromolecules 1987, 20, 2226-2237.

(36) Ajji, A.; Carreau, P. J.; Schreiber, H. P.; Rudin, A. J. Polym. Sci, Polym. Phys. Ed. 1986, 24, 1983-1990.

(37) Kotliar, A. M.; Kumar, R.; Back, R. A. J. Polym. Sci., Polym. Phys. Ed. 1990, 28, 1033-1045.

(38) Teng, C.; Gao, Y.; Wang, X.; Jiang, W.; Zhang, C.; Wang, R.; Zhou, D.; Xue, G. Macromolecules 2012, 45, 6648-6651.

(39) Brown, H. R.; Wignall, G. D. Macromolecules 1990, 23, 683685.

(40) McGrath, K. J.; Roland, C. M.; Weiss, R. G. Macromolecules 1993, 26, 6127-6128.

(41) Wang, X. L.; Tao, F. F.; Sun, P. C.; Zhou, D. S.; Wang, Z. Q.; Gu, Q.; Hu, J. L.; Xue, G. Macromolecules 2007, 40, 4736-4739. 